\title{
Landau-Kleffner Syndrome
}

National Cancer Institute

\section{Source}

National Cancer Institute. Landau-Kleffner Syndrome. NCI Thesaurus. Code C84806.

A rare childhood syndrome characterized by the progressive or sudden inability to understand and use spoken language (aphasia) and paroxysmal electrical brain waves.

Patients develop epileptic seizures and behavioral changes. 